\title{
Attentional Modulation of Learning-Related Repetition Attenuation Effects in Human Parahippocampal Cortex
}

\author{
Do-Joon Yi and Marvin M. Chun \\ Department of Psychology, Yale University, New Haven, Connecticut 06520
}

Two of the most fundamental processes in biological vision are attention and learning. Attention actively selects and enhances visual information that is most relevant to behavior. Learning enables the visual system to benefit from perceptual experience. The amount of visual information to learn is infinite; however, top- down control mechanisms must somehow regulate learning to achieve an adaptive balance between plasticity and stability in neural circuitry. Functional magnetic resonance imaging (fMRI) can measure learning-related changes in neural activity to previously viewed perceptual stimuli. Described variably as the repetition suppression or adaptation effect, the attenuation in neural activity to repeated stimuli versus novel stimuli provides a marker for stimuli-specific perceptual processing and memory. One important issue concerns whether repetition attenuation is automatic or not, and recent work has begun to show that it is sensitive to task demands. Accordingly, the present study further examined how attention controls the attenuated response to repeated stimuli, specifically testing whether attention is important for initial encoding, for the expression of memory traces, or for both encoding and expression. To manipulate attention, we used overlapping scene and face images and asked subjects to attend to either category. fMRI revealed significant attenuation in the parahippocampal place area for only the repeated scenes that were attended both during the initial presentation and during repetition. Thus, attention actively governs when neuronal activity is attenuated to repeated perceptual input, and such attention is important during both initial encoding and subsequent expression of the learned information.

Key words: attention; perceptual learning; memory; repetition attenuation; fMRI; parahippocampal cortex

\section{Introduction}

An essential finding for the study of perceptual memory is that the functional magnetic resonance imaging ( $\mathrm{fMRI}$ ) signal is typically attenuated to "old" repeated stimuli compared with novel stimuli (Squire et al., 1992; Buckner et al., 1995, 1998; GrillSpector and Malach, 2001; Maccotta and Buckner, 2004). This attenuation effect in human fMRI studies has a well documented physiological basis. Single-cell recordings in nonhuman primates indicate that populations of neurons show less activity after repeated exposure to objects and faces than during the initial, novel presentation of these stimuli (Baylis and Rolls, 1987; Brown et al., 1987; Li et al., 1993). The reduction in activity reflects "adaptive filtering," based on a proposal that learning causes pruning of cells that poorly represent the features of a stimulus, resulting in a smaller, but more selective, population of cells (Desimone, 1996; Wiggs and Martin, 1998). Alternatively, attenuation may occur if the entire population of selective neurons exhibits dampened or a shorter duration of activity to old images (Henson and Rugg, 2003). In either case, the attenuation effect can be interpreted as a signature of perceptual memory for previously viewed

Received Nov. 15, 2004; revised Feb. 28, 2005; accepted March 1, 2005.

This work was supported by National Institutes of Health Grant EY014193. We thank Russell Epstein and Daphne Bavelier for helpful discussions that motivated some of our manipulations. We also thank Min-Shik Kim for suggesting the task-irrelevant change trials. David Widders gratefully assisted in running the experiments.

Correspondence should be addressed to Dr. Marvin M. Chun, Department of Psychology, Yale University, P.O. Box 208205, 2 Hillhouse Avenue, New Haven, CT 06520. E-mail: marvin.chun@yale.edu.

D0I:10.1523/JNEUROSCI.4677-04.2005

Copyright $\odot 2005$ Society for Neuroscience $\quad$ 0270-6474/05/253593-08\$15.00/0 stimuli across intervals of seconds, minutes, and even days (Fahy et al., 1993; van Turennout et al., 2000).

The attenuation effect was originally considered to be automatic, but recent studies indicate that the attenuation effect can be actively modulated by behavioral goals and attention (Henson et al., 2002; Dobbins et al., 2004; Eger et al., 2004; Ishai et al., 2004; Murray and Wojciulik, 2004). This raises fundamental questions concerning how attention regulates the learning-related attenuation effect in neural circuitry.

The first question examines which stage of perceptual encoding and memory that attention is important for. Attention may be required only for encoding a novel stimulus during initial presentation, or attention may only be required for the expression (retrieval) of the memory traces. Alternatively, attention may be required for both encoding and expression. Experiment 1 tests the requirement of attention for the initial presentation and repetition of stimuli in the learning-related repetition attenuation effect.

Second, previous studies have used stimulus displays in which attended and ignored items were spatially segregated (Eger et al., 2004) or separable using spatial attention (Murray and Wojciulik, 2004). Thus, spatial selection mechanisms may be required to filter unattended items from being learned. To test whether attention may control neural attenuation independent of spatial locus, we used overlapping face-scene images that could not be segregated using spatial attention mechanisms alone (O'Craven et al., 1999).

The final issue concerns whether previous demonstrations of 
attentional modulation simply reflect floor effects (Henson et al., 2002; Dobbins et al., 2004; Eger et al., 2004; Ishai et al., 2004). Because withdrawing attention away from a stimulus reduces the overall neural response, attenuation effects may simply scale with the overall level of activity, such that attenuation would not be observed whenever any type of manipulation reduces the overall level of activity and corresponding blood-oxygenation-leveldependent (BOLD) contrast signal (Avidan et al., 2002). Experiment 2 tests the more intriguing hypothesis that attenuation effects remain under top-down control at signal strength levels comparable with that observed when stimuli are unattended.

\section{Materials and Methods}

We conducted two fMRI experiments. Our analyses were focused on scene-related activity in the parahippocampal place area (PPA), which was independently localized in individual subjects. The PPA responds strongly to scenes and negligibly to faces (Epstein and Kanwisher, 1998; Epstein et al., 1999), allowing us to probe attentional modulation of neural scene learning that was not confounded by face presentation. Importantly, the PPA signal is sensitive to attentional modulation as well as repetition of scenes (O'Craven et al., 1999; Epstein et al., 2003; Yi et al., 2004). Accordingly, experiment 1 repeated scenes across trials while the scenes were attended or ignored. To distinguish attentional engagement in different learning stages, a factorial design manipulated attention during initial presentation and separately during repetition. Experiment 2 replicated the attentional modulation of learning with overall PPA signal levels controlled across the attended and unattended conditions.

Subjects. Sixteen normal subjects (eight females, three left handed; mean age, 20 years old; range, $18-25$ years old) participated in experiment 1 , and an additional 16 subjects were newly recruited in experiment 2 (seven females, one left handed; mean age, 22 years old; range, 19-32 years old). The study protocol was approved by the Yale University Institutional Review Board, and informed consent was obtained from the subjects.

Experiment 1. Subjects performed a change-detection task for a sequence of two composite scene-face stimuli, attending to either scenes or faces, which were either identical or not within each trial. Treating each trial sequence of two composite stimuli as a single event, we focused our fMRI analyses on the trials in which no change occurred in either the scene or face. To measure repetition attenuation effects, we repeated the scenes across separate trials. Thus, repetition attenuation was defined as the difference between the PPA signal to the trial in which the scene appeared for the first time ("the initial presentation trial") compared with the PPA signal elicited by the subsequent trial in which the same scene appeared again ("the repetition trial").

There were eight runs with each run consisting of 23 event-related change-detection task trials. Every trial presented a sequence of two 533 ms overlapping scene and face composite images, separated by a $767 \mathrm{~ms}$ blank interval (Fig. 1a,b). The perception of an overlapping scene and face was generated by presenting the two stimuli in rapid alternating flicker such that they replaced each other every $33.3 \mathrm{~ms}$ in the center of the screen. The flicker helped promote clearly segregated percepts for each type of image in the same overlapping location. All stimuli, faces and scenes, were $7 \times 7^{\circ}$ gray scale real pictures presented against a gray background. A black-outlined white disk was placed $1^{\circ}$ above the center (between the two eyes of the face) as a fixation mark, subtending $0.1^{\circ}$ in diameter. The first and last pictures of each flicker sequence were a blackand-white checkerboard (element size, $0.7 \times 0.7^{\circ}$ ) to signal the beginning and the end of each alternation.

All stimuli consisted of these overlapping scene-face images, which supported two types of attention conditions, blocked separately: the attend-scene condition and the attend-face condition. In the attendscene blocks, subjects were instructed to detect a possible change of the scene across the two composite images within each trial, while ignoring a possible change of the face. In contrast, in the attend-face block, subjects searched for face changes while ignoring possible scene changes. At the beginning of each block, subjects were cued by a phrase ("attend scene" or "attend face") in yellow for $3 \mathrm{~s}$, followed by a $1 \mathrm{~s}$ blank period. At the end of each trial, a "same different" cue prompted subjects to make a response within $2 \mathrm{~s}$. If the pictures of the attended category were the same, subjects pressed a button with the index finger. Otherwise, they pressed another button with the middle finger. After the response, the fixation mark turned into either a yellow " $\mathrm{S}$ " (for the attend-scene block) or "F" (for the attend-face block) to remind subjects of the type of category they should attend to. The fixation letter remained on the screen until the next trial, which was signaled by blinking the fixation off and on for $400 \mathrm{~ms}$ each. The intertrial interval was $16 \mathrm{~s}$.

As described previously, some of the scenes were repeated across two separate trials. Apart from the within-trial repetition for the changedetection task, the across-trial repetitions were incidental to subjects and served as the events to measure repetition attenuation effects. The attenuation effect for across-trial repetitions of scenes was based on only those trials in which the two composite scene-face images were identical within the trial sequence ("same" response trials for the change-detection task, whichever category was attended) (Fig. 1b). Importantly, when repeated across trials, each of these scenes was paired with a different face. Thus, the associated neural adaptation should be accounted for by the repetition of scenes between trials, not just low-level features.

Attentional modulation of scene-specific learning was measured by comparing the activations of repeated scenes across trials as a function of whether the scene was attended or ignored during the initial presentation trial or the repetition trial. Within each run, eight scenes were repeated as the primary trials for analysis (Fig. 1c). During initial presentation, four of the eight scenes were attended in an attend-scene block [novelattended scene condition (NewAtt)], whereas the other four scenes were ignored in an attend-face block [novel-ignored scene condition (NewIgn)]. Then, by varying whether the scenes repeated within or across interleaving blocks, our design yielded four types of repeated scenes with distinct attentional histories: (1) scenes attended initially and attended during repetition (AttAtt); (2) scenes ignored initially and ignored during repetition (IgnIgn); (3) scenes attended initially but ignored during repetition (AttIgn); and (4) scenes ignored initially but attended during repetition (IgnAtt). Thus, attentional modulation of scene learning can be measured as a reduction in the PPA response to the four types of repeated scenes compared with the PPA response to their initial (novel) presentations. Repetitions occurred across two (32 s) trials with $25 \%$ probability or three ( $48 \mathrm{~s}$ ) trials with $75 \%$ probability. There were two trials for each attentional history condition in each run.

Each run had one of two types of block orderings: face-scene-face or scene-face-scene. In addition to the eight critical trials described above, we included a number of filler trials that were excluded from the analyses. The first trial of each block was not used for the critical trials because of possible task-switching confounds. Intermixed throughout the run were (1) four filler trials that presented a task-relevant change (e.g., face change in an attend-face block), for which subjects were supposed to respond "different"; (2) two filler trials in which the task-irrelevant picture changed (e.g., scene change in an attend-face block), so that subjects should respond "same" because the task-relevant image did not change; and (3) one filler trial in which (1), (2), or no change occurred randomly in each run. The trials with task-irrelevant change were included to motivate subjects to attend selectively to either category. Because we did not analyze the face responses per se (to ensure sufficient power for the scene manipulations), we allowed faces to be reused four times across eight runs, but never within two subsequent runs. Scenes were never reused across runs. Feedback was given verbally after each run.

Right after the change-detection task scans, subjects performed a scene recognition test in the scanner without brain image acquisition. In each of 64 trials, two scenes were presented on each side of the screen; one was among the 64 (eight critical scenes $\times$ eight runs) critical scenes presented during the change-detection task, and the other was novel. The position of each was randomized. Subjects made a two-alternative forced choice in an unspeeded manner regarding which scene had been presented during the change-detection task.

The final two runs were devoted to functional localization of the PPA. The design consisted of alternating blocked presentation of faces and scenes, with each block containing 20 images presented for $800 \mathrm{~ms}$ followed by a blank period of $200 \mathrm{~ms}$. There were nine blocks each of faces and scenes in one fMRI run. Subjects searched for consecutive repetitions 


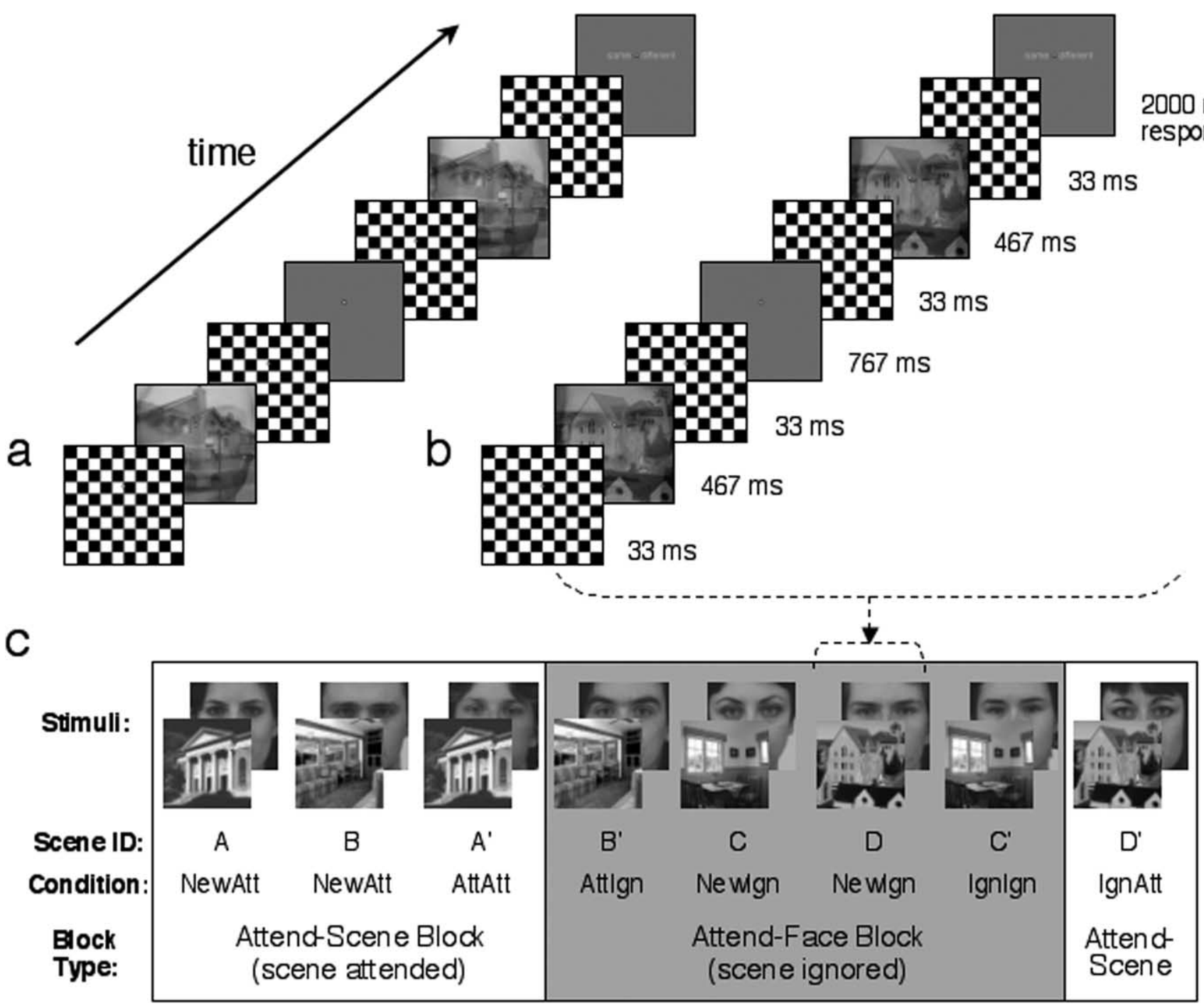

Figure 1. Schematic trial and the design of experiment 1. On each trial, subjects performed a change-detection task for a sequence of two composite scene-face stimuli, attending to either scenes or faces (see Materials and Methods). Each trial sequence was followed by a fixation period (not shown in this figure), in which a fixation point turned into " $\mathrm{S}$ " in the attend-scene block or " $\mathrm{F}$ " in the attend-face block. The sequence of two composite stimuli was treated as a single event in all fMRl analyses. $\boldsymbol{a}$, Example of a filler trial with the face changed. In the attend-face block, the change was task relevant, and thus the correct response was "different." In the attend-scene block, in contrast, the change was task irrelevant, and thus the correct response was "same." $\boldsymbol{b}$, Example of the primary trials, in which change never happened. Whichever category was attended, the correct response was "same." Such trials without any change were the focus of our fMRI analyses. $\boldsymbol{c}$, Illustration of the experimental design. Each scene-face pair stands for one of the primary trials, in which two identical composite stimuli were presented in rapid sequence as shown in $\boldsymbol{b}$. Here, the scene and face image were spatially segregated to improve their visibility for illustration purposes, showing that scenes were repeated across two separate trials, each paired with a different face. During the initial presentation, novel scenes were either attended (e.g., A and B) or ignored (C and D), providing two attention-modulated "new" scene conditions (NewAtt, novel attended; Newlgn, novel ignored). Likewise, when these scenes were subsequently repeated across trials, they were either attended $\left(A^{\prime}\right.$ and $\left.D^{\prime}\right)$ or ignored $\left(B^{\prime}\right.$ and $\left.C^{\prime}\right)$, providing four old conditions with different attention histories (AttAtt, attended and attended; Attlgn, attended and ignored; IgnAtt, ignored and attended; Ignlgn, ignored and ignored).

of stimuli, with two such repetitions occurring in each block. Each face and scene image appeared once per run.

Experiment 2. To equate overall PPA response levels for the attended and unattended conditions, we blurred scenes in the attend-scene block but not those in the attend-face block. The degraded perceptual quality of the blurry scenes reduced the PPA response compared with intact scenes, as demonstrated previously for face-related activity with blurry faces (Vuilleumier et al., 2003). To achieve a constant level of perceptual difficulty across subjects, the blurriness of attended scenes was adjusted in a scene-discrimination task before scanning. Overlapping face-scene images (using rapid alternating flicker) were presented, and subjects were asked to tell whether the scene was indoor or outdoor while ignoring the face. The SD of a Gaussian low-pass filter $\left(1.75 \times 1.75^{\circ}\right)$ was initially $0.2^{\circ}$ and then decreased by $0.01^{\circ}$ until subjects made three correct responses of four consecutive trials (mean SD, $0.14^{\circ}$ ).

During functional scans, subjects first performed four runs of the change-detection task. Each run consisted of an attend-scene block followed by an attend-face block, or vice versa. Each block had 12 eventrelated trials. In the attend-scene block, all scenes were blurred with a Gaussian low-pass filter. In contrast, scenes were fully intact in the attend-face block, as in experiment 1.

Each run presented eight randomly selected scenes for the critical trials to be analyzed (Fig. 2). Four scenes were repeated while attended during the attend-scene block, and the other four were repeated while ignored during the attend-face block. The mean time elapsed between scene repetitions was $42.7 \mathrm{~s}$ for both repeated scene conditions. The design had four conditions from two factors: attention (attended vs ignored scenes) and history (novel vs repeated scenes). Intermixed throughout the run were (1) four filler trials that presented a task-relevant change; (2) two filler trials in which the task-irrelevant picture changed; and (3) two filler trials in which (1), (2), or no change occurred randomly in each run. Other protocols and methods were the same as in experiment 1. 
Subjects subsequently performed a scene recognition test of 32 trials (eight critical scenes $\times$ four scan runs) as in experiment 1 . When the blurry scenes in the attend-scene blocks were tested, both the old scenes (target) and novel scenes (distractor) were low-pass filtered to the same extent as in the changedetection task. The scene recognition test was followed by one or two PPA localizer runs.

fMRI acquisition. A $3 \mathrm{~T}$ Trio scanner (Siemens, Erlangen, Germany) was used with a standard birdcage head coil. T2* scan parameters were as follows: time to repeat, $2 \mathrm{~s}$; time to echo, $25 \mathrm{~ms}$; flip angle, $80^{\circ} ; 3.75 \times 3.75 \times 7 \mathrm{~mm}$ voxel size. Stimuli were presented through a liquid-crystal display projector onto a rear projection screen located at the feet of the subjects and viewed with angled mirrors. Responses were collected with an MRI-compatible button box. Each run in experiment 1 acquired 186 image volumes (195 in experiment 2), each with 19 axial slices ( $7 \mathrm{~mm}$ thick, no skip) parallel to the anterior commissure-posterior commissure line.

$f M R I$ analyses. The first six image volumes were discarded to allow for T1 equilibration. Images for each subject were realigned to correct for movement using SPM99 (Wellcome Department of Cognitive Neurology, Institute of Neurology, London, UK). Analyses were then conducted with the Yale fMRI software package (http://mri.med.yale.edu/ fmri_software.htm).

Our analyses were focused on the PPA as a region of interest (ROI), and post hoc whole-brain analyses did not reveal significant activity for the comparisons of interest beyond the PPA in both of our experiments. The PPA was first localized in each individual by contrasting the averaged brain activity in scene blocks with face blocks. Statistical parametric maps of BOLD activation for each subject were created using a skew-corrected percentage of signal difference. The PPA ROI was defined as the voxel with the peak activation and its eight surrounding voxels, such that each subject provided a $3 \times 3$ voxel grid from each hemisphere (Marois et al., 2004; Yi et al., 2004). For all subjects, the activated region was found in the parahippocampal gyrus/collateral sulcus region, consistent with previous studies (mean Talairach coordinates: $x=28, y=-44, z=-9 ; x=$ $-27, y=-48, z=-8$ in experiment $1 ; x=27, y=-47, z=-9 ; x=$ $-29, y=-47, z=-7$ in experiment 2) (Levy et al., 2001; Epstein et al., 2003).

From each PPA ROI of individual subjects, the time course of percentage of signal change from the first volume after trial onset was extracted for each condition. Time courses were collapsed between both hemispheres and averaged across all subjects ( post hoc analyses did not reveal any significant effect of hemisphere or any interaction with the other factors). Following previous convention (Epstein et al., 2003; Marois et al., 2004; Yi et al., 2004), statistical analyses (ANOVA and paired $t$ tests) were performed on the fourth volumes after trial onset (peristimulus time, $6 \mathrm{~s}$ ) as the peak amplitude response. Area-under-the-curve analyses confirmed the peak analyses for the critical pairwise comparisons below.

\section{Results}

\section{Experiment 1}

During the scanning session, subjects detected task-relevant changes better in the attend-scene condition than in the attendface condition (Fig. 3a, hit rates) (99 vs 81\%). Error rates followed the same patterns; the false alarm rates were lower in the attend-scene condition than in the attend-face condition for no change trials ( 1 vs $2 \%$ ) as well as for task-irrelevant change trials (catch error rates; 5 vs 45\%). This performance difference between attention conditions may be attributable to the fact that scenes are more heterogeneous than faces, so that scene feature changes were more salient than face feature changes. The behavioral and neural data below indicate that the attention manipu- a

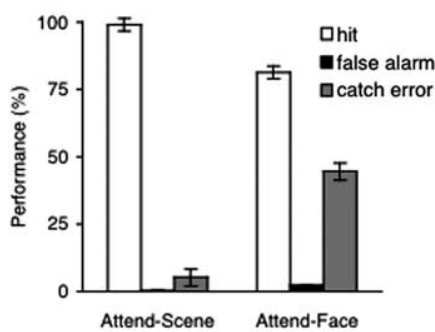

C
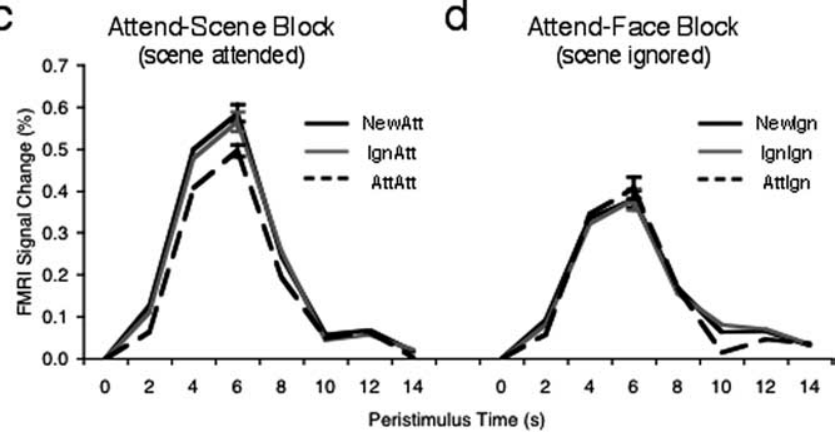

Figure 3. Results of experiment 1. $\boldsymbol{a}$, Change-detection performance. $\boldsymbol{b}$, Scene-recognition performance. Note that all scenes were attended once more during the recognition test. The dashed line indicates the $50 \%$ chance level. Asterisks indicate above-chance recognition. $\boldsymbol{c}, \boldsymbol{d}$, Activations in the PPA ROI. The error bars indicate \pm SEM.

lation was highly effective. After excluding incorrect responses, the mean time elapsed between across-trial scene repetitions was $45.2 \mathrm{~s}$ without any differences among the four repeated scene conditions (all $t_{(15)}<2.9$; all $p>0.1$ ).

In the behavioral scene recognition test, subjects recognized more attended scenes with performance lowest for scenes that were never attended (Fig. 3b). The scenes that were attended at least once were recognized better than chance (all $t_{(15)}>8.7$; all $p<0.001$ ), whereas the scenes that were only ignored (IgnIgn) were recognized no better than chance $\left(t_{(15)}=1.307 ; p>0.1\right)$. The scenes that were always attended (AttAtt) were recognized best (from other conditions; all $t_{(15)}>2.8$; all $p<0.05$ ).

Attentional modulation was also evident in the PPA signal changes. Novel scenes produced greater PPA activation in the 
attend-scene condition than in the attend-face condition, showing that attention enhanced perceptual processing (O'Craven et al., 1999) [Fig. 3, $c$ (NewAtt) vs $d$ (NewIgn) $\left(_{(15)}=6.114 ; p<\right.$ 0.0001 for the peak).

To examine the effects of attention on the repetition suppression effect, the peak signals were submitted to a 2 (attention: attended vs ignored scenes) $\times 3$ (history: previously attended, previously ignored, and novel scenes) repeated-measures ANOVA. Significant effects of attention $\left(F_{(1,15)}=37.257 ; p<\right.$ $0.001)$ and an interaction between attention and history $\left(F_{(2,30)}=\right.$ 4.873; $p<0.05)$ were revealed. The effect of history failed to reach significance $\left(F_{(1,15)}=1.065 ; p>0.1\right)$.

A priori comparisons directly compared attenuation effects across the different attentional histories. We compared the activation for old repeated scenes to the activation for novel scenes. Because of significant differences in PPA activation to novel scenes as a function of attention, it is critically important to use the appropriate baseline, comparing ignored repeated trials with ignored novel trials and attended repeated trials with attended novel trials. A significant repetition suppression effect was observed only for the scenes that were attended both during initial presentation and during repetition (AttAtt; $t_{(15)}=4.710 ; p<$ $0.001)$. No repetition suppression was observed when the scenes were ignored during both the initial presentation and repetition trials (IgnIgn; $t_{(15)}=0.026 ; p>0.1$ ). Moreover, attention during initial exposure alone (AttIgn) or during the repetition alone (IgnAtt) was not sufficient to observe the suppression effect (both $t_{(15)}<0.9$; both $p>0.1$ ). These findings suggest that neural adaptation requires attention both during the encoding of new perceptual experience and during the expression of past experience.

\section{Experiment 2}

Was the lack of attenuation in the face task of experiment 1 simply attributable to the reduced level of activity in the PPA to ignored scenes, a floor effect? To demonstrate that attention is important rather than signal strength per se, we conducted a second experiment in which the overall activation to attended scenes was reduced to the level of that for the ignored scenes. We achieved this by blurring the scenes in the attend-scene blocks (Vuilleumier et al., 2003). Because low spatial frequencies still carry the scene spatial layout information (Schyns and Oliva, 1994; Oliva and Schyns, 1997), scene-specific activity should be preserved when the scenes are attended. If attention is critical, then repetition attenuation should occur for attended blurry scenes. If overall signal strength is important, then the attenuation effect should be abolished for attended blurry scenes. No attenuation should be observed for ignored intact scenes, replicating our first experiment.

Subjects performed change detection better in the attendscene condition than in the attend-face condition (hit rates; 98 vs $88 \%$, respectively) (Fig. $4 a$ ). Error rates showed the same patterns; the false-alarm rates were lower in the attend-scene condition than in the attend-face condition for no change trials (1 vs $4 \%$ ) as well as for task-irrelevant change trials (catch error rates; 5 vs $61 \%)$.

In the scene-recognition test, attended blurry scenes were recognized at better than chance (Fig. $4 b$, AttAtt) $\left(t_{(15)}=3.297 ; p<\right.$ $0.01)$, whereas ignored intact scenes were not (IgnIgn; $t_{(15)}=$ $1.440 ; p>0.1)$.

Novel blurry scenes in the attend-scene condition produced low PPA activation that was comparable with novel intact scenes in the attend-face condition [Fig. 4, $c$ (NewAtt) vs $d$ (NewIgn)] a

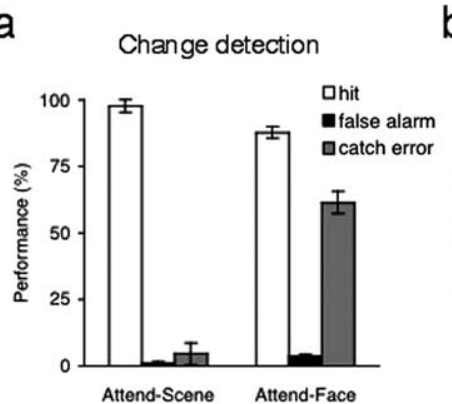

b

Scene recognition

C

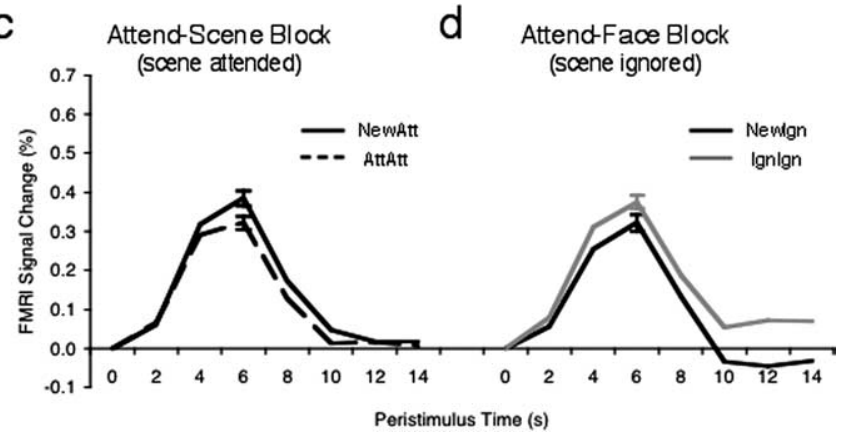

Figure 4. Results of experiment 2. $\boldsymbol{a}$, Change-detection performance. $\boldsymbol{b}$, Scene-recognition performance. The dashed line indicates the $50 \%$ chance level. The asterisk indicates abovechance recognition. $\boldsymbol{c}, \boldsymbol{d}$, Activations in the PPA ROI. The error bars indicate \pm SEM.

$\left(t_{(15)}=1.737 ; p>0.1\right)$ (also compare with NewIgn in Fig. $3 d$ ), indicating successful control for overall signal strength. Nevertheless, even at this lower BOLD contrast signal level, attended blurry scenes produced significantly lower activation during repetition than during initial presentation (AttAtt; $t_{(15)}=2.545 ; p<$ $0.05)$. In contrast, ignored intact scenes failed to produce significant attenuation during repetition, in fact bordering repetition enhancement (IgnIgn; $\left.t_{(15)}=1.995 ; p=0.065\right)$.

A 2 (attention: attended vs ignored) $\times 2$ (history: repeated vs novel) repeated-measures ANOVA showed only a significant interaction between attention and history $\left(F_{(1,15)}=10.818 ; p<\right.$ $0.01)$. Neither attention nor history showed a significant effect (both $F_{(1,15)}<0.1$; both $\left.p>0.7\right)$. When we considered experiments as a between-subject factor ("experiment") and confined analysis to scenes repeated within a block (AttAtt and IgnIgn), a mixed design ANOVA revealed a significant effect of attention and a significant interaction between attention and experiment (all $F_{(1,30)}>15$; all $\left.p<0.01\right)$. Moreover, the interaction between attention and history was significant $\left(F_{(1,30)}=15.074 ; p<0.01\right)$, which, importantly, did not interact with experiment (no threeway interaction; $F_{(1,30)}=0.263 ; p>0.6$ ), demonstrating attentional modulation of repetition attenuation in both experiments. All other effects failed to show significance $\left(\right.$ all $F_{(1,30)}<4$; all $p>$ 0.1 ). Overall, our results indicate that the lack of attenuation in inattention conditions is not simply attributable to lower levels of activity but rather reflect the more fundamental modulatory effects of attention.

\section{Discussion}

This study demonstrated that attention modulates learningrelated attenuation activity in the PPA for repeated scenes. Thus, the repetition attenuation effect is not automatically driven by stimulus exposure alone but is modulated by task demands and attention. This finding has ecological value because of the need to balance stability and plasticity in neural circuitry (Grossberg, 
1980). Only attended regularities may induce plasticity and learning. The present findings emphasize a direct role for visual attention, adding to a growing body of studies showing that neural attenuation is modulated by task manipulations (Dobbins et al., 2004; Eger et al., 2004; Ishai et al., 2004; Murray and Wojciulik, 2004; Yi et al., 2004).

An important feature of the present design was its ability to dissociate the effects of attention during initial encoding and during subsequent expression to explore whether attention may be important for only one of those two stages. The results indicate that attention is critical for both initial encoding and for the expression of learning during repetition. The use of overlapping images in this study demonstrate that the attentional selection can be based on objects (O'Craven et al., 1999), suggesting that spatial selection mechanisms may not be required for the attentional modulation of learning.

Our second experiment demonstrates that the absence of attenuation effects during inattention cannot be attributed to floor effects. Because attenuation effects dissipate at very low signal strengths (Avidan et al., 2002), it was critical to rule out such floor effects as an explanation for the lack of attenuation during inattention. Here, attenuation effects only occurred for attended repetitions even when the overall level of BOLD contrast signal was matched across attended and unattended stimulus conditions. Thus, attention was the important mechanism, not the level of neural activity as measured by the fMRI BOLD contrast signal. This provides novel empirical support for modeling work, suggesting that the lack of attenuation effects during inattention conditions is not an artifact of reduced levels of neural activity (i.e., changes in "gain") (Murray and Wojciulik, 2004).

These findings illuminate the tight relationship between attention and the repetition attenuation effect. The results also raise interesting new insights as well as questions about the relationship between the attenuation effect and learning and about the nature of attentional control of such learning effects in neural circuitry.

\section{Repetition attenuation and perceptual memory}

Repetition attenuation effects in neural activity have commonly been interpreted as reflecting stimulus-specific perceptual memory, especially when the attenuation effects occur in cortical regions known to be important for stimulus-specific perceptual processing (Schacter and Buckner, 1998; Henson, 2003). What is not clear is how to characterize the type of memory that the attenuated signal supports. Memory researchers distinguish between explicit, declarative memory and implicit, nondeclarative memory (Tulving and Schacter, 1990; Squire et al., 1993). Explicit memory supports conscious recognition and recollection of facts and events, whereas implicit memory is typically shown with indirect measures such as priming, in which previous experience facilitates a behavioral task even in the absence of conscious recognition of the repeated stimulus.

Although different neural circuitry is involved in performing explicit memory tasks and implicit memory tasks, it is possible that the representations of perceptual stimuli may be common across both explicit and implicit memory. In our study, the attenuation signals in the PPA were associated with subsequent explicit memory performance in the recognition task. However, previous studies have typically associated attenuation signals with implicit memory performance using priming measures (Buckner et al., 1998; Koutstaal et al., 2001; Maccotta and Buckner, 2004). Preliminary work in our own laboratory has also confirmed an association between attenuation in the PPA with be- havioral priming in a scene-matching task requiring speeded responses. Thus, repetition attenuation effects may be associated with both explicit and implicit learning and memory of perceptual events.

With respect to explicit memory, attention may strengthen the encoding of stimuli to be later recognized or recalled. PPA activity was significantly higher for attended scenes than for unattended scenes, consistent with previous work showing that subsequently remembered items are associated with higher activity in parahippocampal and prefrontal regions during encoding (Brewer et al., 1998; Wagner et al., 1998). Although attention may determine the overall level of activity during initial encoding, attenuation may be observed as encoding demands decrease as any given stimulus is repeated (Kirchhoff et al., 2000). However, one must always exercise great caution in interpreting overall BOLD signal strength because it is influenced by so many different factors such as attention, learning, and stimulus/task selectivity.

Another complication is that attenuation effects may reflect the influence of two separate mechanisms. One is a responselearning mechanism that maps perceptual stimuli with specific actions. A recent study showed that neural attenuation only occurred for repeated stimuli that also afforded an identical (repeated) response. Repeated visual objects that were associated with a different response on repetition did not produce neural attenuation effects (Dobbins et al., 2004). Our study may support this response-learning framework because attenuation did not occur for any unattended stimuli that had no response associated with their initial presentation or repetition. Nevertheless, response learning cannot be the sole mechanism because there are cases of repetition attenuation for repeated stimuli that required no response (Henson et al., 2000; Yi et al., 2004) or even orthogonal responses (Henson et al., 2003).

Accordingly, there must be a separate mechanism that is sensitive to perceptual repetition. This may reflect neuronal populations becoming selectively tuned with learning (Desimone, 1996; Wiggs and Martin, 1998), or repetition attenuation may occur when an entire population of selective neurons exhibits a dampened response or a shorter duration of activity to old images (Henson and Rugg, 2003). Together, these various studies point to a distinction between response specificity and stimulus specificity in memory (Schacter et al., 2004). How the two types of specificity interact represents an exciting area for future research (Henson, 2003; Schacter et al., 2004).

This study focused on repetition attenuation effects, but repetition can also lead to enhancement effects. Enhancement appears to occur when repetition is correlated with enhanced recognition of a highly degraded, ambiguous, or very novel stimulus (Dolan et al., 1997; George et al., 1999; Grill-Spector et al., 2000; Henson et al., 2000). However, repetition attenuation effects are the norm when highly visible, familiar stimuli and their associated tasks are repeated.

\section{Mechanisms of attentional control}

The present study demonstrated that attention is important for both initial encoding and subsequent access to perceptual memory traces, but more work is needed to describe the specific nature of the top-down signals that control plasticity in the visual cortex. An attractive candidate mechanism is working memory, which maintains templates of attended stimuli within the frontal and parietal cortex (Desimone and Duncan, 1995). Top-down signals of visual events maintained in working memory may gate whether attenuation effects occur or not. A serious complication 
for this hypothesis is that the attenuation effects in the temporal cortex readily occur even when working memory is occupied by other stimuli (Miller and Desimone, 1994; Yi et al., 2004). Thus, working memory mechanisms may not be involved for on-line control of neural attenuation in the perceptual cortex. Instead, selection may occur within the perceptual cortex proper in the form of biased competition (Desimone and Duncan, 1995) that may not require on-line maintenance of attended events in working memory. The system can be preset to favor one category/task over another, and this may enable plasticity for stimuli of that category alone to the exclusion of the unattended category.

For any manipulation of attention, one must avoid distinguishing attended and unattended conditions in dichotomous terms. Rather, the amount of attention to a stimulus should be described as a continuous variable, such that decreasing attention to a stimulus will lead to decreasingly smaller and less reliable effects on visual learning. In other words, it remains possible that task-irrelevant stimuli may be learned in certain situations (Watanabe et al., 2001; Jiang and Leung, 2005). Specifically, attenuation effects occur for unattended stimuli when the primary task is not sufficiently demanding (Murray and Wojciulik, 2004; Yi et al., 2004), allowing attention to spillover to task-irrelevant stimuli (Rees et al., 1997). The point here is that attention clearly modulates the robustness of learning-related attenuation effects when the perceptual demands on attention are sufficiently taxing to exclude residual processing of unattended items (Eger et al., 2004; Yi et al., 2004).

\section{Summary}

Although questions remain, the present study and others permit several important conclusions. First, attention modulates learning-related attenuation effects, and attention seems to be important for both encoding and expression. Second, the lack of attenuation for unattended stimuli cannot be attributed to floor effects. Third, attentional modulation of repetition attenuation does not require spatial selection; it can be specific to a perceptual category and even to the specific task that one is performing. Finally, working memory is not directly required to observe attenuation, whereas some form of attentional competition within the relevant perceptual stages of analysis may govern whether repetition attenuation occurs or not. These findings provide a strong base to advance our future understanding of the relationship between top-down attentional control mechanisms and learning-related attenuation effects in the perceptual cortex.

\section{References}

Avidan G, Harel M, Hendler T, Ben-Bashat D, Zohary E, Malach R (2002) Contrast sensitivity in human visual areas and its relationship to object recognition. J Neurophysiol 87:3102-3116.

Baylis GC, Rolls ET (1987) Responses of neurons in the inferior temporal cortex in short term and serial recognition memory tasks. Exp Brain Res 65:614-622.

Brewer JB, Zhao Z, Desmond JE, Glover GH, Gabrieli JD (1998) Making memories: brain activity that predicts how well visual experience will be remembered. Science 281:1185-1187.

Brown MW, Wilson FA, Riches IP (1987) Neuronal evidence that inferomedial temporal cortex is more important than hippocampus in certain processes underlying recognition memory. Brain Res 409:158-162.

Buckner RL, Petersen SE, Ojemann JG, Miezin FM, Squire LR, Raichle ME (1995) Functional anatomical studies of explicit and implicit memory retrieval tasks. J Neurosci 15:12-29.

Buckner RL, Goodman J, Burock M, Rotte M, Koutstaal W, Schacter D, Rosen B, Dale AM (1998) Functional-anatomic correlates of object priming in humans revealed by rapid presentation event-related fMRI. Neuron 20:285-296.
Desimone R (1996) Neural mechanisms for visual memory and their role in attention. Proc Natl Acad Sci USA 93:13494-13499.

Desimone R, Duncan J (1995) Neural mechanisms of selective visualattention. Annu Rev Neurosci 18:193-222.

Dobbins IG, Schnyer DM, Verfaellie M, Schacter DL (2004) Cortical activity reductions during repetition priming can result from rapid response learning. Nature 428:316-319.

Dolan RJ, Fink GR, Rolls E, Booth M, Holmes A, Frackowiak RS, Friston KJ (1997) How the brain learns to see objects and faces in an impoverished context. Nature 389:596-599.

Eger E, Henson RN, Driver J, Dolan RJ (2004) BOLD repetition decreases in object-responsive ventral visual areas depend on spatial attention. J Neurophysiol 92:1241-1247.

Epstein R, Kanwisher N (1998) A cortical representation of the local visual environment. Nature 392:598-601.

Epstein R, Harris A, Stanley D, Kanwisher N (1999) The parahippocampal place area: recognition, navigation, or encoding? Neuron 23:115-125.

Epstein R, Graham KS, Downing PE (2003) Viewpoint-specific scene representations in human parahippocampal cortex. Neuron 37:865-876.

Fahy FL, Riches IP, Brown MW (1993) Neuronal activity related to visual recognition memory: long-term memory and the encoding of recency and familiarity information in the primate anterior and medial inferior temporal and rhinal cortex. Exp Brain Res 96:457-472.

George N, Dolan RJ, Fink GR, Baylis GC, Russell C, Driver J (1999) Contrast polarity and face recognition in the human fusiform gyrus. Nat Neurosci 2:574-580.

Grill-Spector K, Malach R (2001) fMR-adaptation: a tool for studying the functional properties of human cortical neurons. Acta Psychol (Amst) 107:293-321.

Grill-Spector K, Kushnir T, Hendler T, Malach R (2000) The dynamics of object-selective activation correlate with recognition performance in humans. Nat Neurosci 3:837-843.

Grossberg S (1980) How does a brain build a cognitive code? Psychol Rev 87:1-51.

Henson R, Shallice T, Dolan R (2000) Neuroimaging evidence for dissociable forms of repetition priming. Science 287:1269-1272.

Henson RN (2003) Neuroimaging studies of priming. Prog Neurobiol 70:53-81.

Henson RN, Rugg MD (2003) Neural response suppression, haemodynamic repetition effects, and behavioural priming. Neuropsychologia 41:263-270.

Henson RN, Shallice T, Gorno-Tempini ML, Dolan RJ (2002) Face repetition effects in implicit and explicit memory tests as measured by fMRI. Cereb Cortex 12:178-186.

Henson RN, Goshen-Gottstein Y, Ganel T, Otten LJ, Quayle A, Rugg MD (2003) Electrophysiological and haemodynamic correlates of face perception, recognition and priming. Cereb Cortex 13:793-805.

Ishai A, Pessoa L, Bikle PC, Ungeleider LG (2004) Repetition suppression of faces is modulated by emotion. Proc Natl Acad Sci USA 101:9827-9832.

Jiang Y, Leung AW (2005) Implicit learning of ignored visual context. Psychon Bull Rev, in press.

Kirchhoff BA, Wagner AD, Maril A, Stern CE (2000) Prefrontal-temporal circuitry for episodic encoding and subsequent memory. J Neurosci 20:6173-6180.

Koutstaal W, Wagner AD, Rotte M, Maril A, Buckner RL, Schacter DL (2001) Perceptual specificity in visual object priming: functional magnetic resonance imaging evidence for a laterality difference in fusiform cortex. Neuropsychologia 39:184-199.

Levy I, Hasson U, Avidan G, Hendler T, Malach R (2001) Center-periphery organization of human object areas. Nat Neurosci 4:533-539.

Li L, Miller EK, Desimone R (1993) The representation of stimulusfamiliarity in anterior inferior temporal cortex. J Neurophysiol 69:1918-1929.

Maccotta L, Buckner RL (2004) Evidence for neural effects of repetition that directly correlate with behavioral priming. J Cogn Neurosci 16:1625-1632.

Marois R, Yi D-J, Chun MM (2004) The neural fate of consciously perceived and missed events in the attentional blink. Neuron 41:465-472.

Miller EK, Desimone R (1994) Parallel neuronal mechanisms for shortterm memory. Science 263:520-522.

Murray SO, Wojciulik E (2004) Attention increases neural selectivity in the human lateral occipital complex. Nat Neurosci 7:70-74. 
O'Craven KM, Downing PE, Kanwisher N (1999) fMRI evidence for objects as the units of attentional selection. Nature 401:584-587.

Oliva A, Schyns PG (1997) Coarse blobs or fine edges? Evidence that information diagnosticity changes the perception of complex visual stimuli. Cognit Psychol 34:72-107.

Rees G, Frith CD, Lavie N (1997) Modulating irrelevant motion perception by varying attentional load in an unrelated task. Science 278:1616-1619.

Schacter DL, Buckner RL (1998) Priming and the brain. Neuron 20:185-195.

Schacter DL, Dobbins IG, Schnyer DM (2004) Specificity of priming: a cognitive neuroscience perspective. Nat Rev Neurosci 5:853-862.

Schyns PG, Oliva A (1994) From blobs to boundary edges: evidence for time and spatial scale dependent scene recognition. Psychol Sci 5:195-200.

Squire LR, Ojemann JG, Miezin FM, Petersen SE, Videen TO, Raichle ME (1992) Activation of the hippocampus in normal humans: a functional anatomical study of memory. Proc Natl Acad Sci USA 89:1837-1841.

Squire LR, Knowlton B, Musen G (1993) The structure and organization of memory. Annu Rev Psychol 44:453-495.
Tulving E, Schacter DL (1990) Priming and human memory systems. Science 247:301-306.

van Turennout M, Ellmore T, Martin A (2000) Long-lasting cortical plasticity in the object naming system. Nat Neurosci 3:1329-1334.

Vuilleumier P, Armony JL, Driver J, Dolan RJ (2003) Distinct spatial frequency sensitivities for processing faces and emotional expressions. Nat Neurosci 6:624-631.

Wagner AD, Schacter DL, Rotte M, Koutstaal W, Maril A, Dale AM, Rosen BR, Buckner RL (1998) Building memories: remembering and forgetting of verbal experiences as predicted by brain activity. Science 281:1188-1191.

Watanabe T, Nanez JE, Sasaki Y (2001) Perceptual learning without perception. Nature 413:844-848.

Wiggs CL, Martin A (1998) Properties and mechanisms of perceptual priming. Curr Opin Neurobiol 8:227-233.

Yi D-J, Woodman GF, Widders D, Marois R, Chun MM (2004) Neural fate of ignored stimuli: dissociable effects of perceptual and working memory load. Nat Neurosci 7:992-996. 